\title{
THE DIFFEOMORPHISM GROUP OF A COMPACT RIEMANN SURFACE
}

\author{
BY C. J. EARLE ${ }^{1}$ AND J. EELLS
}

Communicated by S. Smale, February 6, 1967

1. Introduction. In this note we announce two theorems. The first describes the homotopy type of the topological group $D(X)$ of diffeomorphisms ( $=C^{\infty}$-diffeomorphisms) of a compact oriented surface $X$ without boundary. The second, of which the first is a corollary, gives a fundamental relation among $D(X)$, the space of complex structures on $X$, and the Teichmüller space $T(X)$ of $X$. We make essential use of the theory of quasiconformal mappings and Teichmüller spaces developed by Ahlfors and Bers [3], [6], and the theory of fibrations of function spaces. Our results confirm a conjecture of Grothendieck [7, p. 7-09], relating the homotopy of $D(X)$ and $T(X)$.

2. The theorems. The surface $X$ has a unique (up to equivalence) $C^{\infty}$-differential structure. Let $D(X)$ denote the group of orientation preserving diffeomorphisms. With the $C^{\infty}$-topology (uniform convergence of all differentials) $D(X)$ is a metrizable topological group [8]. We let $D_{0}\left(X ; x_{1}, \cdots, x_{n}\right)$ denote the subgroup of $D(X)$ consisting of those diffeomorphisms $f$ which are homotopic to the identity and satisfy $f\left(x_{i}\right)=x_{i}(1 \leqq i \leqq n)$, where $x_{1}, \cdots, x_{n}$ are distinct points of $X$. This second condition is fulfilled vacuously if $n=0$.

THEOREM 1. Let $g$ denote the genus of $X$.

(a) If $g=0$, then $D_{0}\left(X ; x_{1}, x_{2}, x_{3}\right)$ is contractible. Furthermore, $D(X)$ is homeomorphic to $G \times D_{0}\left(X ; x_{1}, x_{2}, x_{3}\right)$, where $G$ is the group of conformal automorphisms of the Riemann sphere.

(b) If $g=1$, then $D_{0}\left(X ; x_{1}\right)$ is contractible. Furthermore, $D_{0}(X)$ is homeomorphic to $G \times D_{0}\left(X ; x_{1}\right)$, where now $G$ is the identity component of the group of conformal automorphisms of the torus.

(c) If $g \geqq 2$, then $D_{0}(X)$ is contractible.

Corollary. In all cases $\mathfrak{D}_{0}(X)$ is the identity component of $D(X)$.

Remark 1. Part (a) is equivalent to the theorem of Smale [9] asserting that the rotation group $\mathrm{SO}(3)$ is a strong deformation retract of $D\left(S^{2}\right)$. Our proof is entirely different from Smale's.

REMARK 2. A concept of differentiability has recently been de-

\footnotetext{
1 Research partially supported by NSF Grant GP6145.
} 
veloped by J. A. Leslie, relative to which he has shown that the group $\mathscr{D}(X)$ - for any compact differential manifold-is a Lie group [8].

Next, let $M(X)$ denote the $C^{\infty}$-complex structures on $X$; with the $C^{\infty}$-topology this is a convex open subset of a Fréchet space. Any subgroup of $\mathfrak{D}(X)$ operates (on the right) on $M(X)$ in the obvious way. If $g \geqq 1$ let $T(X)$ denote the Teichmüller space of $X$. For the definition of that space we refer to [2], [6, Lecture 1]; however, Theorem 2 below provides a new characterization.

Theorem 2. Let $g$ denote the genus of $X$.

(a) If $g=0$, then $D_{0}\left(X ; x_{1}, x_{2}, x_{3}\right)$ is homeomorphic to $M(X)$.

(b) If $g=1$, then $D_{0}\left(X ; x_{1}\right)$ operates principally (i.e., continuously, freely, properly, and with local sections) on $M(X)$. The quotient space is (homeomorphic to) $T(X)$.

(c) If $g \geqq 2$, then $D_{0}(X)$ operates principally on $M(X)$. The quotient space is (homeomorphic to) $T(X)$.

3. On the proof of Theorem 1. We proceed to indicate how Theorem 1 is derived from Theorem 2 .

First of all, $M(X)$ is always contractible. Therefore Theorem $2 \mathrm{a}$ easily implies Theorem 1a. Secondly, the quotient map $M(X) \rightarrow T(X)$ defines a principal fibre bundle. A fundamental theorem of Teichmüller (see [1], [5] for efficient proofs) asserts that $T(X)$ is a finite dimensional cell. Thus from the homotopy sequence of our fibration we conclude that all the homotopy groups of the structural groups $D_{0}\left(X ; x_{1}\right)$ and $\mathscr{D}_{0}(X)$ vanish. Finally, these structural groups are metrizable manifolds modeled on Fréchet spaces; therefore, they are absolute neighborhood retracts. By a theorem of J. H. C. Whitehead the vanishing of their homotopy groups implies their contractibility. Theorem 1 follows.

4. On the proof of Theorem 2. For simplicity of exposition we consider only the cases $g \geqq 2$. We represent $X$ as the quotient of the upper half plane $U$ by a Fuchsian group $\Gamma$ operating freely on $U$. The $C^{\infty}$ complex structures ( $=C^{\infty}$ Beltrami differentials [2]) on $X$ are represented by the $C^{\infty}$ complex valued functions $\mu$ on $U$ satisfying

$$
\mu(\gamma z) \overline{\gamma^{\prime}(z)} / \gamma^{\prime}(z)=\mu(z)
$$

and $\max \{|\mu(z)|: z \in U\}<1$. Each such function $\mu$ determines uniquely a diffeomorphism $f: U \rightarrow U$ such that

$$
\mu(z)=\mu_{f}(z)=f_{\bar{z}}(z) / f_{z}(z),
$$

and $f$ leaves the points $0,1, \infty$ fixed. 
The group $D_{0}(X)$ is identified with the group of diffeomorphisms $h: U \rightarrow U$ which commute with all elements of $\Gamma$. The action of $D_{0}(X)$ on $M(X)$ is given by

$$
\mu_{f} \cdot h=\mu_{f_{0} h} .
$$

This action is principal, and the quotient $M(X) / \mathscr{D}_{0}(X)$ is homeomorphic to $T(X)$. For the verification we first study the dependence on $\mu$ of the solutions of Beltrami's equation $f_{\bar{z}}=\mu f_{z}$. Next we verify that $M(X) / \mathfrak{D}_{0}(X)$ maps bijectively and continuously onto $T(X)$. Finally, a theorem of Ahlfors and Weill [4] provides us with local sections from $T(X)$ into $M(X)$.

\section{REFERENCES}

1. L. V. Ahlfors, On quasiconformal mappings, J. Analyse Math. 4 (1954), 1-58, 207-208.

2. - Teichmüller spaces, Proc. Internat. Congr. Math. 1962, Institute Mittag-Leffler, Djursholm, Sweden, 1963.

3. - Lectures on quasiconformal mappings, Mathematical Studies No. 10, Van Nostrand, Princeton, N. J., 1966.

4. L. V. Ahlfors and G. Weill, A uniqueness theorem for Beltrami equations, Proc. Amer. Math. Soc. 13 (1962), 975-978.

5. L. Bers, "Quasiconformal mappings and Teichmüller's theorem," in Analytic functions, pp. 89-119, Princeton Univ. Press, Princeton, N. J., 1960.

6. - On moduli of Riemann surfaces, Lecture notes at Eid. Tech. Hoch. Zürich (1964).

7. A. Grothendieck, Techniques de construction en gêométrie analytique, Séminaire H. Cartan, Ecole Norm. Sup., Paris (1960/61) Expose 7-8.

8. J. A. Leslie, On a differential structure for the group of diffeomorphisms, Topology (to appear).

9. S. Smale, Diffeomorphisms of the 2-sphere, Proc. Amer. Math. Soc. 10 (1959), 621-626.

Cornell University AND

Churchill College, Cambridge 